\title{
A SURVEY ON APPLICATIONS OF CLOUDLET INFRASTRUCTURE IN MOBILE CLOUD COMPUTING
}

\author{
Srilatha.M ${ }^{1}$, S.Rajeshwari ${ }^{2}$ and K.Rani ${ }^{3}$
}

Abstract:Mobile cloud computing (MCC) integrates the cloud computing into the mobile environment to improve the performance (e.g., battery life, storage, and bandwidth), environment (e.g., scalability, and availability), and security (e.g., reliability and privacy). The cloudlet is one of the mobile cloud computing infrastructures to reduce power and network delays. In this paper we presented different application and research proposals of cloudlets.

Key words: Mobile cloud computing, cloudlet.

MCC is emerging research area that combines Cloud Computing technology and the mobile environment to create new infrastructure that is capable of performing intensive computations and storing massive amount of data[1]. The mobile device users are provided with an online access to an unlimited computing power and storage space as shown in fig 1 .

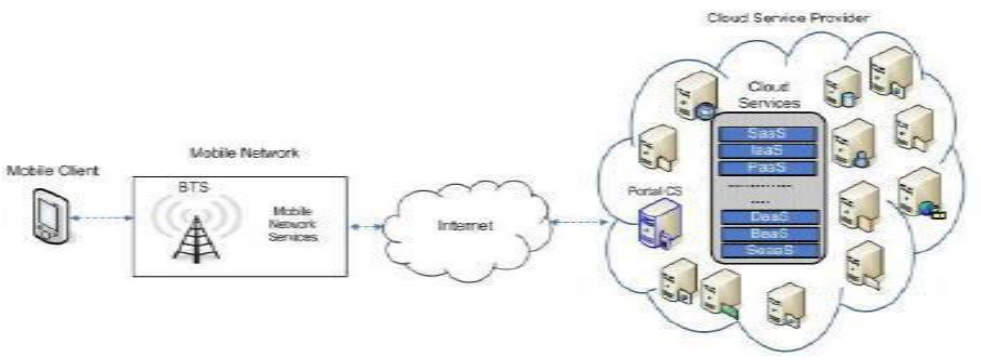

Fig 1.

Cloudlet is resource-collection of computers which is well-connected to the Internet and available for use by nearby mobile devices. Hence, when mobile devices cannot or do not want

${ }^{1}$ Deaprtment of Computer Science, VR Siddhartha Engineering College Andhrapradesh, India

${ }^{2}$ Deaprtment of Computer Science, VR Siddhartha Engineering College Andhrapradesh, India

${ }^{3}$ Deaprtment of Computer Science, VR Siddhartha Engineering College Andhrapradesh, India 
to connect to the cloud, they can find and access a nearby computing resource. In this way mobile device offloads its workload to a local 'cloudlet' comprised of several multi-core computers with connectivity to the remote cloud servers. . A cloudlet is similar to a small data center that is located on designated areas/places and is connected to a larger cloud server via the Internet.

Cloudlets refer to the infrastructure consisted of local physical equipments attach to different department or unit, inside the cloud computing based information system [5]. These infrastructures are distributed on location but stay at the same level logically as shown in figure 2 .

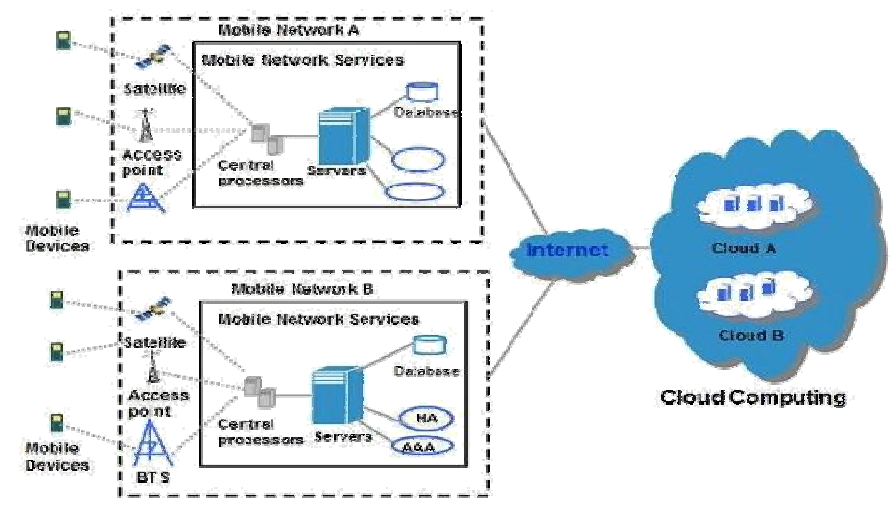

Fig 2.

In paper [2] e presented efficient techniques to reduce energy consumption in MCC using Offloading, green MCC, Cloudlet architecture, and clone.

In paper [3] authors are implemented MOCHA (mobile-cloudlet-cloud architecture) for face recognition by partitioning the computation (tasks) among the available cloud servers and the cloudlet. This can be done in teo ways (1) Fixed: the tasks are equally distributed among the available cloud servers (or the cloudlet). (2) Greedy: based on cloud servers response times task are allocated to the server (cloudlet) that can complete the task in the minimum amount of time. Authors implemented Cloud-Vision face recognition application perform the face recognition for a mobile device.It is is executed in two separate phases: 1) face detection using Viola-Jones algorithm that progressively narrows an initial large set of face location candidates to the final set of detected face locations; and 2) face recognition, which uses the classic Eigenfaces approach, to determine the likelihood of each detected face matching one of the template images in a database. These computation can be partitioned among mulitple cloud servers to speed up the response time.

In paper [4] focused on an optimal admission control policy for MCC. There is no standard mechanism to charge users based on their usage of services by service providers. Hence authors proposed an optimization model for allocating resources of bandwidth and cloudlet to meet the requirement of mobile users based on the semi-Markov decision process (SMDP). Here the 
policy of SMDP obtained by transforming an original optimization problem into a linear programming (LP). In this processes an admission control mechanism of the MCC hotspot is designed to decide whether an arriving request from mobile user can be accepted or not. It takes the state of MCC hotspot into account and makes decision according to the optimal policy to be obtained from SMDP.

When Given a set of users operating battery-powered devices and requesting different types of cloud services and a set of cloudlets then power consumption may be more. In paper [6] suggested a solution to assign each user request to a specific cloudlet capable to service by minimizing the overall power consumption. The power consumption was minimized by examining that whether the user is in coverage region of cloudlet at particular time slot or not and then applied a cost function to represent total power consumption of the system.

In paper [7] focused on impact of cloudlets on interactive mobile cloud applications (IMCA) such as file editing, video streaming and chatting with mobile nodes moving from one cloudlet coverage to another cloudlet coverage area. To evaluate performance in IMCA authors are implemented a hybrid ad-hoc-like wireless routing protocol by modifying the DestinationSequenced Distance-Vector routing (DSDV) ad-hoc routing protocol in NS2. Then compared experimental result with pure cloud computing communication. Finally concluded that using cloudlet data transfer delay will be reduced and throughput will be increased.

In article [8] specified that a mobile user exploits virtual machine (VM) technology to customize service software on a nearby cloudlet (like in fig.) and then uses that service over a wireless LAN. To deploy cloudlet like wi-fi it is needed to simplify cloudlet management for integrating Wi-fi with cloudlet .In order to do this, authors introduced a solution called transient customization of cloudlet infrastructure using hardware VM technology. It emphasis on pre-use customization and post-use cleanup ensure that cloudlet infrastructure is restored to its pristine software state after each use, without manual intervention.

[9] In paper presented a novel cloudlet-based efficient data collection system in Wireless Body Area Networks (WBANs). Wireless Body Area Networks (WBAN) consists of a group of communicating sensor nodes, which can monitor different body parameters and gather a lot of body information. These devices, communicating through wireless technologies, can transmit data from the body to a base station from where the data can be forwarded to a hospital, clinic, or a service provider in real-time manner as shown in fig 3. The huge amount of data collected by WBAN nodes demands scalable, on-demand, powerful, and secures storage and processing infrastructure.

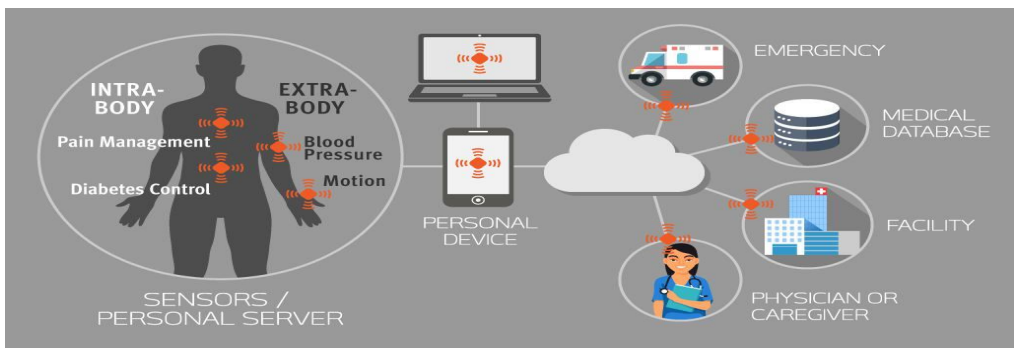

Fig 3. 
So authors proposed a prototype of WBANs, including Virtual Machine and Virtualized Cloudlet. It had been evaluated using extended CloudSim simulator. Using this prototype users or service providers can accesses data by minimizing end-to-end packet cost and packet-to-cloud energy.

In paper [10] proposed an analytical approach for performance analysis and modeling of cloudlet called interacting stochastic sub-models. In this processes, different servicing steps of cloudlet architecture mapped into separate stochastic sub-models. These sub-models interact with each other such that the outputs of ones are transmitted to the other as inputs and vice versa. By iteration over this process the final results will be obtained. These stochastic sub-models are named as Request Admission Engine(RAE), Host selection engine(HSE), Cloudlet provisioning engine (LPE) and public cloud provisioning Engine (PPE)) that each of which covers the behavior of appropriate queue and its corresponding engine.

In paper [11], proposed pocket cloudlets, an effective architecture that leverages abundant nonvolatile memories(NVM )in mobile devices to significantly improve user experience, both in terms of latency and battery life, by avoiding expensive radio wakeups and transmissions to access cloud services. The problems with mobile cloud services are like the amount of data to be stored locally on the device, a mechanism to manage the locally stored cloud data, storage architecture for efficiently storing and accessing this large amount of data.

To solve these problems authors implemented PocketSearch, a mobile search pocket cloudlet that lives on the phone and is able to answer queries locally without having to use the $3 \mathrm{G}$ link 2 . It consists of two components: the community and the personalization components. The community part of the cache is responsible for storing the small set of queries and search results that are popular across all mobile users. This information is automatically extracted from the search $\operatorname{logs}$ and is updated overnight every time the mobile device is recharging. The personalization part of the cache monitors the queries entered as well as the search results clicked by the user and performs two discrete tasks. First, it expands the cache to include all those queries and search results accessed by the user that did not initially exist in the community part of the cache. Second, it collects information about user clicks, such as when and how many times the user clicks on a search result after a query is submitted, to customize ranking of search results to user's click history. When a query is submitted, PocketSearch will first perform a lookup in the cache to find out if there are locally available search results for the given query. In the case of a cache hit, the search results are fetched from the local storage, ranked based on the past user access patterns recorded by the personalization part of the cache, and immediately displayed to the user. In the case of a cache miss, the query is submitted to the search engine over the $3 \mathrm{G}$ radio link.

In paper [12], authors analyzed the computation offloading problem in cloudlet-based mobile cloud computing. They worked on the computation offloading strategy of multiple users via multiple wireless APs using a game-theoretic solution. It uses two algorithms with respect to heterogeneous and homogenous mobile users. The performance of this algorithm is compared 
with two other computation offloading strategies:-1) Local Computation: all mobile users execute their computation tasks locally on their mobile devices. 2) Random Selection: mobile users either randomly chooses one of the wireless APs to offload their computation tasks or execute their tasks locally.

In paper[13], authors proposed an advanced dynamic model, dynamic energy-aware cloudletbased mobile cloud computing model (DECM), that leverages cloudlets technique to assign, manage, and optimize the cloud-based infrastructure usages and services for achieving green computing. This model uses dynamic programming to assist cloudlets to determine the cloud computing resources within a changing operational environment. Where cloud lets are conceptualize as a dynamic cloudlet (DCL) to achieve following missions:

Determining and predict physical machines on the cloud using dynamic programming

Calculations of simple applications and quickly respond to end users.

Predict whether users should switch to other cloudlets and enable a real-time suggestion for cloudlets switching.

The scenario of this algorithm is that the mobile device connects to the cloud server by one route M1 at the beginning of the service implementation. Moving forward to the next time unit, a decision will be made depending on the comparison among all routes. The route(s) with a better performance will be selected. The resolution will be made for each time unit by using the same method and finally reach a minimum total energy cost with quality services in a defined timing period. This approach was efficient in a specific condition that implies the users can accept a scalable service delivery manner during the usages.

In paper [14] proposed a cloudlet-based multi-lateral resource exchange framework for mobile users, relying on no central entities. In this paper proposed a generic mobile cloud computing framework (with underlying technology BitCoin) where all types of underlying resources can be exposed to the mobile users through virtualization. According to this processes a Cloudlet is deployed by a user provide services to the other mobile users as shown in fig 4.

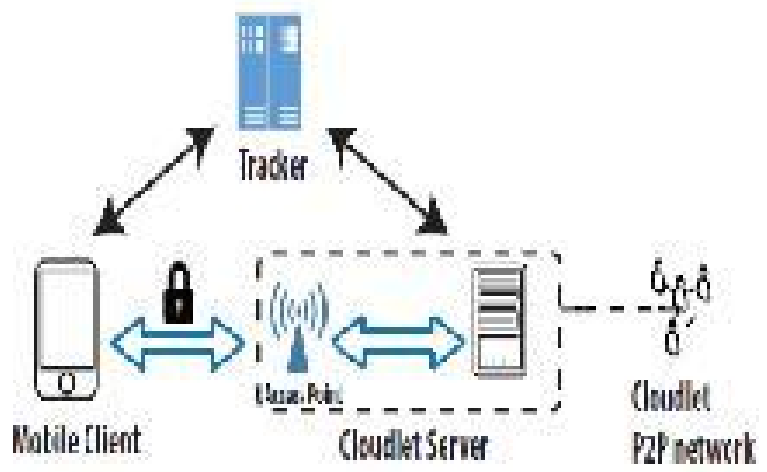

Fig 4. 
To check the effectiveness of this framework, authors were also implemented a prototype enabling Internet bandwidth leasing among mobile user through the users cloudlet servers. This had done by splitting energy-consuming tasks to the cloudlet side, while keeping necessary userinteractive tasks on the mobile side.

The connections between a mobile user and mobile cloudlets can be intermittent because of mobility and cloudlet capacity. As a result, offloading actions taken by the mobile user may fail. So in paper [15], authors developed an optimal offloading model Markov decision process (MDP) based dynamic offloading algorithm for the mobile user to intermittently connect cloudlet system, by considering the users' local load and availability of cloudlets. The algorithm is designed as follows

During the application execution, assumed a time interval $\mathrm{t}$ for the user to finish executing any application phase locally or remotely on cloudlets.

At the beginning of each decision period, the user observes the current system states, i.e., the number of jobs $Q$ in the queue, the application phase $G$ that the user is executing, and the number of accessible cloudlets $\mathrm{N}$.

Based on the observed composite state $S=(G, Q, N)$ of the system, the user computes the immediate costs of local execution and offloading

In General cloudlets have limited resources and processing abilities, which implies that they may not be capable to process every incoming request. Instead, some resource-intensive requests need to be sent to remote data centers for processing. In paper [16] addressed the online request admission issue in a cloudlet by maximizing the system throughput. The online request throughput maximization problem is to determine whether an arrival request to be admitted or rejected by the system such that the system throughput is maximized for a specified time period T. The system throughput is the ratio of the number of admitted requests to the number of requests for period $\mathrm{T}$. The algorithm for online request throughput maximization is processed as follows.

1. It first checks whether the requested amount of each resource $\mathrm{r} i, \mathrm{k}(\mathrm{t})$ can be met by the system.

2. If not, the request is rejected immediately;

3. Otherwise the system calculates the admission cost of processing the request based on the load of each resource at this moment.

4. If its admission cost is beyond a specified threshold of each resource in the system, the request will be rejected; otherwise, it is admitted by the cloudlet.

In paper [17] proposed a mechanism to identify a cloudlet for computation offloading in a decentralized manner. It will be dine in two ways.

1. The first phase Mobile device acquires information of the available cloudlets

2. The mobile device now has to select a cloudlet based on the information and requirement of the application to be offloaded. 
If the application requirement related to RAM and CPU is known beforehand, cloudlet specifications given in the Cloudlet structure related to memory and CPU are compared. If the cloudlet doesn't have enough memory, the application may not run with proper performance and hence should not be chosen.

In paper [18] compared the performances of translating various n-grams executed on a cloud and on a cloudlet by using multilingual dictionary application based on the dynamic VM synthesis. It consists of the following elements:

1. A server on which the mobile device sends words or phrases for translation

2. Translation of the words or phrases, and returning appropriate translation back to the mobile device.

If cloudlet is set as a cluster structure, VMs can be easily cloned and thus gain parallelism. The processes of dynamic VM synthesis are shown in fig 5.

\section{Fig 5}

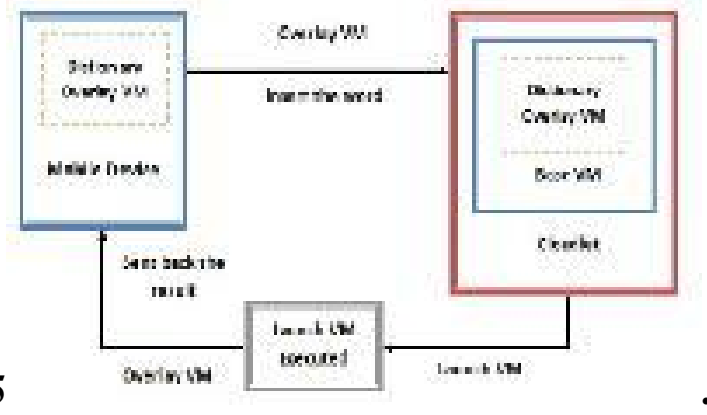

When customer tries to translate any word or phrase, first the mobile device will try to find if there are local LAN cloudlets available. The mobile device is connected to the cloudlet by using high-bandwidth wireless LAN. If there is no cloudlet which can perform such tasks then the mobile application tries to connect to a distant WAN based cloud server which can perform the translation.

In paper[19] presented Personal Cloudlets framework called OPENi's to allows users share, reuse, and control access to their data across many mobile applications by maintaining cloud scalability. OPENi consist of two APIs:

1. The API framework: allows for frictionless interoperability between cloud based services.

2. The Personal Cloudlet framework is a virtual space that securely stores user data and gives users primary control over that data.

Along with above two components it also consists of the other components called mobile client library, the security framework. The architecture of OPENi was described as follows 


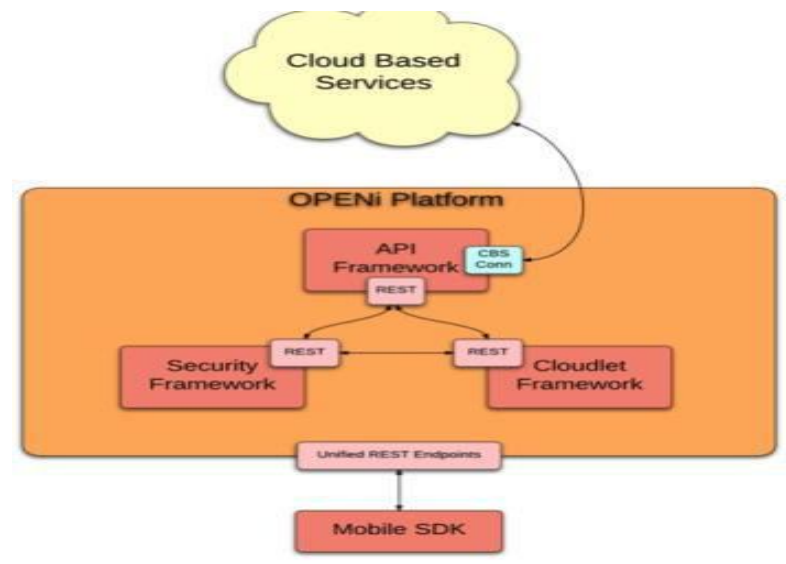

The main objective of this approach is to:

1) To build a web based security and authorization framework that will satisfy the service provider's requirements and a context broker that will enable the sharing of context information between applications in accordance with the users privacy settings.

2) To deliver an open source platform that will allow application consumers to create, deploy and manage their personal space in the cloud (Personal Cloudlet). Each Personal Cloudlet will constitute a novel entity that will be linked to its user's identity over the web in a similar way that a social profile does today.

3) To provide and promote a novel, user-centric application experience of cloud-based services not only across different devices but also inherently across different applications. The OPENi framework will enable application consumers to share and distribute their data across their applications.

4) To ensure the OPENi platform maintains a low barrier to entry for application developers and service providers.

In paper [20] described a distributed system that creates a 3D map of the complete world that is continuously updated by crowd-sourced depth information. In this model, information of system is encoded as a depth map (or point cloud): a set of discrete points with $3 \mathrm{D}$ coordinates. Then the point cloud spited into sub models when it has become too large to ensure fast and accurate registration. Now each submodel is assigned to a different VM. The submodels hosted in the VMs on a cloudlet will contain details of geographically close environments. Users are connected to a nearby cloudlet, the submodel is deployed on the cloudlet to which the user was connected. A central locator component keeps the mapping between geographical coordinates and cloudlet IP. 


\section{REFERENCE}

[1] Niroshinie Fernando, Seng W. Loke , Wenny Rahayu,"Mobile cloud computing": A survey.In Fututre Genetation Computer System 29(2013) 84-206.

[2] Khadijah S. Bahwaireth, Lo'ai Tawalbeh, Anas Basalamah, Yaser Jararweh Mohammad, Tawalbeh, "Efficient Techniques for Energy Optimization in Mobile Cloud Computing": 978-1-5090-0478-2/15/\$31.00 @2015 IEEE.

[3] Soyata T, Muraleedharan R, Funai C, Kwon M, Heinzelman W. Cloud-vision, "Realtime face recognition using a mobile-cloudlet-cloud acceleration architecture": IEEE symposium on computers and communications (ISCC). IEEE; 2012.p.059-66.DOI: http://dx.doi.org/10.1109/ISCC.2012.6249269.

[4] Hoang DT, Niyato D, Wang P,"Optimal admission control policy for mobile cloud computing hotspot with cloudlet": IEEE wireless communications and networking conference (WCNC)" IEEE;2012. p. 3145- 9. DOI: http://dx.doi.org/10.1109/WCNC.2012.6214347.

[5] Han Xingye, Li Xinming, Liu Yinpeng," Research on Resource Management for Cloud Computing Based Information System": 2010 International Conference on Computational and Information Sciences.

[6] M. Al-Ayyoub, Y. Jararweh, L. Tawalbeh, E. Benkhelifa, and A.Basalamah,"Power Optimization of Large Scale Mobile Cloud Computing Systems":IEEE Fi-Cloud, 3rd International Conference on Future Internet of Things and Cloud, Rome, Italy, 24-28 Aug, (2015).

[7] Fesehaye D, Gao Y, Nahrstedt K, Wang G, "Impact of cloudlets on interactive mobile cloud applications": IEEE 16th international enterprise distributed object computing conference (EDOC). IEEE; 2012. p. 123-32. DOI: http://dx.doi.org/10. 1109/EDOC.2012.23.

[8] Satyanarayanan M, Bahl P, Caceres R, Davies N,"The case for vm-based cloudlets in mobile computing": IEEE Pervasive Compute 2009;8(4):14- 23.http://dx.doi.org/10.1109/MPRV.2009.82.

[9] Quwaider M, Jararweh Y.," Cloudlet-based efficient data collection in wireless body area networks": Simulation Model Practice and Theory 2015; 50:57-71. http://dx.doi.org/ 10.1016/j.simpat.2014.06.015.

[10] Raeia , Nasser Yazdani ,Reza Shojaee, "Modeling and Performance Analysis of Cloudlet in Mobile Cloud Computing": Hassan Journal of Performance Evaluation, September, 2016, DOI: http://dx.doi.org/10.1016/j.peva.2016.10.005.

[11]Emmanouil Koukoumidis, Dimitrios Lymberopoulos,"Pocket Cloudlets": Karin Strauss.ASPLOS'11, March 511, 2011, Newport Beach, California, USA, ACM 978-1-4503-0266-1/11/03.

[12] Xiao Ma, Chuang Lin, Xudong Xiang" Game-theoretic Analysis of Computation Offloading for Cloudletbased Mobile Cloud Computing": MSWiM'15, November 2-6, Cancun, Mexico.

[13] Gai K, et al, "Dynamic energy-aware cloudlet-based mobile cloud computing model for green computing": Journal of Network and Computer Applications (2015), http://dx.doi.org/10.1016/j.jnca.2015.05.016i.

[14] Y. Wu, L. Ying,"A Cloudlet-based Multi-lateral Resource Exchange Framework for Mobile Users": proceedings of the IEEE INFOCOM, 2015, pp. 927-935. DOI:10.1109/INFOCOM.2015.7218464.

[15] Y. Zhang, D. Niyato, W. Ping, "Offloading in mobile cloudlet systems with intermittent connectivity":IEEE Transactions on Mobile Computing 14 (12) (2015) 2516-2529. 
[16] ] Q. Xia, W. Liang and W. Xu, "Throughput maximization for online request admissions in mobile cloudlet" :IEEE 38th Conference on Local Computer Networks (LCN 2013), October 2013.

[17] Dilay Parmar, A. Sathish Kumar, Ashwin Nivangune, "Discovery and Selection Mechanism of Cloudlets in a Decentralized MCC environment”: 2016 IEEE/ACM International Conference on Mobile Software Engineering and Systems.

[18] Aleksandar Bahtovski, Katerina Zdravkova, Marjan Gusev, "Performance of Cloudlet-based Multilingual Dictionary”: $2015 \quad$ ACM. ISBN 978-1-4503-3335-1/15/09.DOI:

http://dx.doi.org/10.1145/2801081.2801092.

[19] D’onal McCarthy , Paul Malone, etc, "Personal Cloudlets: Implementing a User-Centric Data store with Privacy Aware Access Control for Cloud-based Data Platforms“:2015 IEEE DOI: 10.1109/TELE RISE.2015.15. 